\title{
Simulated Space VLBI Experiment using the Tidbinbilla 11-m DSN Tracking Station and SURFSAT and Simulated Satellites
}

R. Wietfeldt, J. Smith, J. Border, V. Altunin, \& M. Ryne Jet Propulsion Laboratory, Pasadena CA, U.S.A.

C. Lewis, J. Benson, M. Claussen, \& J. Romney

National Radio Astronomy Observatory, Socorro NM, U.S.A.

J. Springett

NeoComm Systems, La Crescenta CA, U.S.A.

J. Webber

National Radio Astronomy Observatory, Charlottesville VA, U.S.A.

E. King, J. Reynolds, W. Wilson, D. Jauncey, \& R. Ferris

Australia Telescope National Facility, CSIRO, Epping, Australia

P. Harbison \& B. Jenkins

Canberra Deep Space Communications Complex, Canberra, ACT, Australia

M. Costa \& S. Ellingsen

U. of Tasmania, Hobart, Australia

C. Knight

Interferometrics, Fairfax VA, U.S.A.

Abstract. We describe a space VLBI experiment involving an earth-orbiting satellite (SURFSAT) and simulated satellites, a space VLBI ground tracking station, an array of ground radio telescopes, and a space VLBI correlator. The purpose of this experiment was to provide as complete as possible an end-to-end simulation of space VLBI in preparation for the first space VLBI mission, VSOP, and in particular to test the most critical aspect of space VLBI, viz. the ability to generate a stable and accurate frequency standard (clock) for the orbiting VLBI element.

\section{Introduction}

The primary purpose of this experiment was twofold: to test the ability of the DSN 11-m ground tracking stations (GTS), newly constructed for the space VLBI missions VSOP and RadioAstron, to generate a stable and accurate clock for use in space VLBI; and to test the ability of the VLBA correlator to apply the "time corrections" from space VLBI observations, supplied by JPL, to create the stable and accurate satellite clock during the correlation process.

\section{Observations}

In this experiment, one performs "usual interferometry" between ground radio telescopes (GRT), with each GRT driven by its native H-maser frequency reference, but with one modification: one GRT is driven by a frequency reference derived from a reference signal transmitted by a GTS and returned from an orbiting satellite, or a satellite simulated within the GTS. We refer to this 


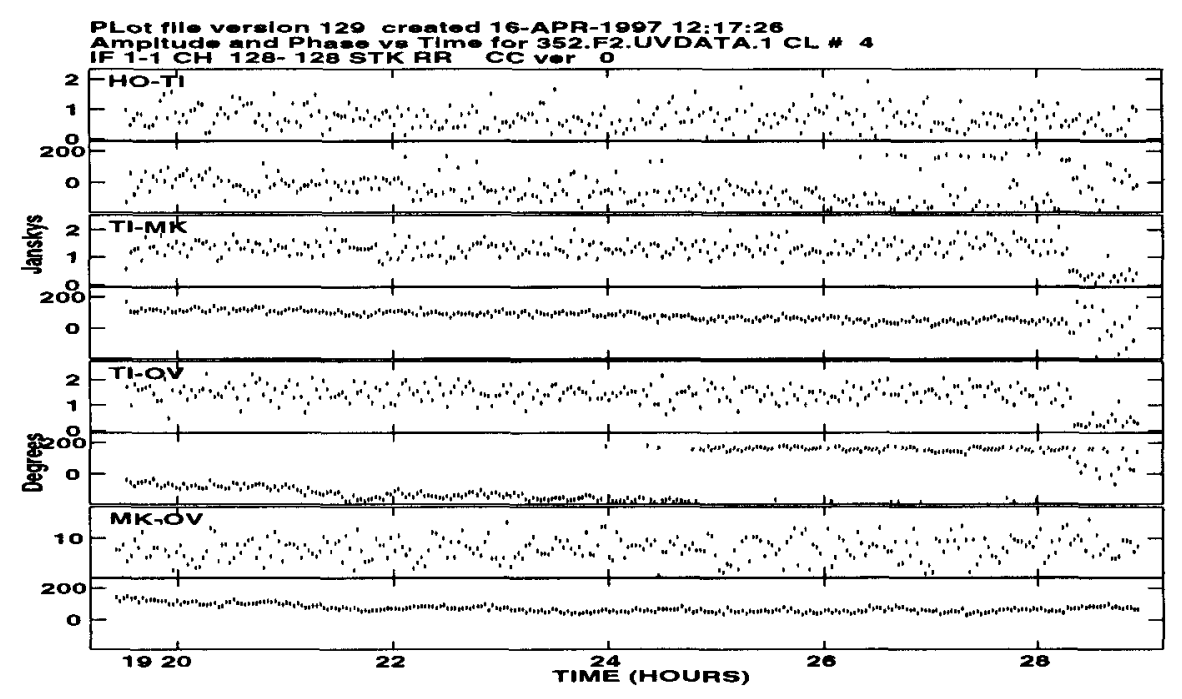

Figure 1. VLBA correlator results from the SURFSAT satellite pass on day 352 , 1996. "Raw" correlator amplitude and phase are shown (no fringe-fitting), after application of the space VLBI time corrections to remove the effects of the two-way phase transfer through the SURFSAT satellite.

frequency reference, corrupted primarily by its transmission path through the atmosphere, or the GTS simulator, as the "dirty" clock, and the GRT using this as its frequency reference the "dirty" GRT. The use of such a frequency standard for one GRT would generally result in the loss of fringes to that station, were it not for the measurement by the GTS of the "phase residual" or difference phase between the uplink and downlink signals, which may be used at the correlator in the form of "time corrections" to correct for the variations in signal phase of the "dirty" reference applied to the "dirty" GRT recordings.

VLBI data were acquired at four GRTs in December 1996: Tidbinbilla (TI) and Hobart (HO) (recorded on S2 recorders, and subsequently tape-copied to VLBA tape format), and VLBA MK and OV (recorded on VLBA recorders). The TI (70-m) antenna served as the "dirty" GRT, driven by the "dirty" clock both from the SURFSAT satellite and simulated space VLBI satellites. Tapes for the four GRTs, now all in VLBA tape format, were shipped to the VLBA correlator and correlated using time correction files created at JPL. The results from a 10-minute SURFSAT pass are shown in Figure 1. The TI "dirty" baselines show no appreciable differences compared to the MK-OV "clean" baseline, demonstrating the effectiveness of the space VLBI time corrections and the general ability of the DSN tracking station and VLBA correlator to support space VLBI observations. Similar results have been achieved using the simulated satellite technique.

Acknowledgments. Part of this work has been undertaken at the Jet Propulsion Laboratory, California Institute of Technology, under contract to the National Aeronautics and Space Administration. The National Radio Astronomy Observatory is a facility of the NSF, operated under a cooperative agreement by Associated Universities, Inc. 